\title{
Botrychium dusenii (H.Christ) Alston (Ophioglossaceae) nueva adición para la flora vascular del Parque Nacional Pali Aike, Región de Magallanes, Chile
}

\section{Botrychium dusenii (H.Christ) Alston (Ophioglossaceae) additions to the flora of Pali Aike National Park, Region of Magallanes, Southern Chile}

\author{
Erwin Domínguez $Z^{1}$, Ángel Suarez ${ }^{1}$, Nelson Bahamonde ${ }^{1} \&$ Sergio Opazo ${ }^{2}$ \\ ${ }^{1}$ Instituto de Investigaciones Agropecuarias - INIA-Kampenaike. Casilla 277, Punta Arenas. Chile. \\ 2 Universidad de Magallanes, Escuela de Ciencias Agropecuarias, Avenida Bulnes 01855, Punta Arenas, Chile. \\ edominguez@inia.cl
}

\begin{abstract}
We report the presence of Botrychium dusenii (H. Christ) Alston (Ophioglossaceae), collected for the first time in the Pali Aike National Park, increasing to two cited pteridophytes for this protected area. This finding extends the distribution range of this species towards the Patagonian Steppe.
\end{abstract}

En la Región de Magallanes se han citado 56 especies de Pteridófitas (Henríquez et al. 1995), 18 de las cuales están catalogadas con problemas de conservación (Rodríguez 1995, Baeza et al. 1998). El género Botrychium está representado en Chile por Botrychium australe R. Br. subsp. negeri (Christ) R.T. Claisen y Botrychium dusenii (H. Christ) Alston (Rodríguez 1995). Esta última se distribuye entre las regiones de Aysén y Magallanes sobre el límite altitudinal del bosque deciduo, formando parte de la vegetación alto andina y del complejo de la tundra magallánica (Pisano 1980).

En la Región de Magallanes se encuentra preferentemente en el sector occidental entre las latitudes $50^{\circ} 45^{\prime}$ hasta $55^{\circ}$ 38'S, creciendo sobre suelo arenoso en el borde costero y en sitios expuestos entre los 0 a $900 \mathrm{~m}$ de altitud (Moore 1983, Arroyo et al. 1992, Domínguez et al. 1999). Una de las características de esta especie es que es posible encontrarla formando agrupaciones de pocos individuos, siendo extremadamente escasa (Looser 1971), clasificada como rara y endémica para el extremo sur de la Patagonia (Arroyo et al. 1992, Baeza et al. 1998, Zuloaga et al. 2010) y como Vulnerable para Chile por Rodríguez et al. (2009). En Chile ha sido citada para las siguientes áreas protegidas: Reserva Nacional Laguna Geinimeni (Saldivia \& Rojas 2006), Parque Nacional Torres del Paine (Pisano 1974, Arroyo et al. 1992), P. N. Alberto María De Agostini (Domínguez et al. 1999, Henríquez 2002) y P. N. Cabo de Hornos (Pisano 1980, Dollenz 1982).

Para el Parque Nacional Pali Aike (PNPA) ha sido citada la presencia de Cystopteris fragilis (L.) Bernh. (Dryopteridaceae) como la única especie perteneciente al grupo de las Petridófitas. Esta área protegida se caracteriza por representar la provincia biótica de la Estepa Patagónica en Chile (Fig. 1a), donde el clima corresponde a Estepa Fría, caracterizado por la presencia de fuertes vientos del oeste, con temperaturas medias de 3 a $4^{\circ} \mathrm{C}$, y precipitaciones anuales entre 200 y $250 \mathrm{~mm}$, distribuidas homogéneamente durante el año (Pisano 1977, Endlicher \& Santana 1988). La vegetación está dominada por Festuca gracillima Hook.f. y F. pallescens (St.-Yves) Parodi, junto a un extenso matorral bajo de Empetrum rubrum Vahl ex Willd. y parches de matorrales de Chiliotrichum diffusum (G.Forst.) Kuntze y Adesmia boronoides Hook.f. Su flora está representada por 164 especies de plantas vasculares (Domínguez et al. 2004). El único cuerpo de agua dulce en el parque es Laguna Ana

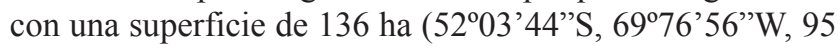
$\mathrm{m}$ s.n.m). La que presenta un progresivo retroceso durante los últimos 25 años, liberando una importante superficie, la que ha sido colonizada por diversas especies hasta hoy poco estudiadas (Figs. $1 \mathrm{~b}$ y $1 \mathrm{c}$ ). El presente trabajo reporta el hallazgo de una población de $B$. dusenii en el sector de la Laguna Ana (PNPA), y proporciona antecedentes sobre el tipo de hábitat donde se encontró esta especie y la flora vascular asociada.

El hallazgo de $B$. dusenii en diciembre de 2010 en el PNPA da cuenta de su condición única en el extremo pluviométrico árido hacia el oriente en la estepa patagónica. De acuerdo a los trabajos disponibles de flora para la 
Región de Magallanes, representa el primer registro en un área ambientalmente tan diferente a las localidades reportadas hasta ahora, aumentando la diversidad de la flora vascular del parque a 165 especies. La vegetación en los márgenes de la laguna Ana se distribuye en forma de anillos, siendo notoriamente diferente del resto del parque (Fig. 2, Tabla I). Se recomienda mantener la exclusión del ganado doméstico en el interior de esta área protegida, condición que ha favorecido a numerosas especies con problemas de conservación dentro del parque.
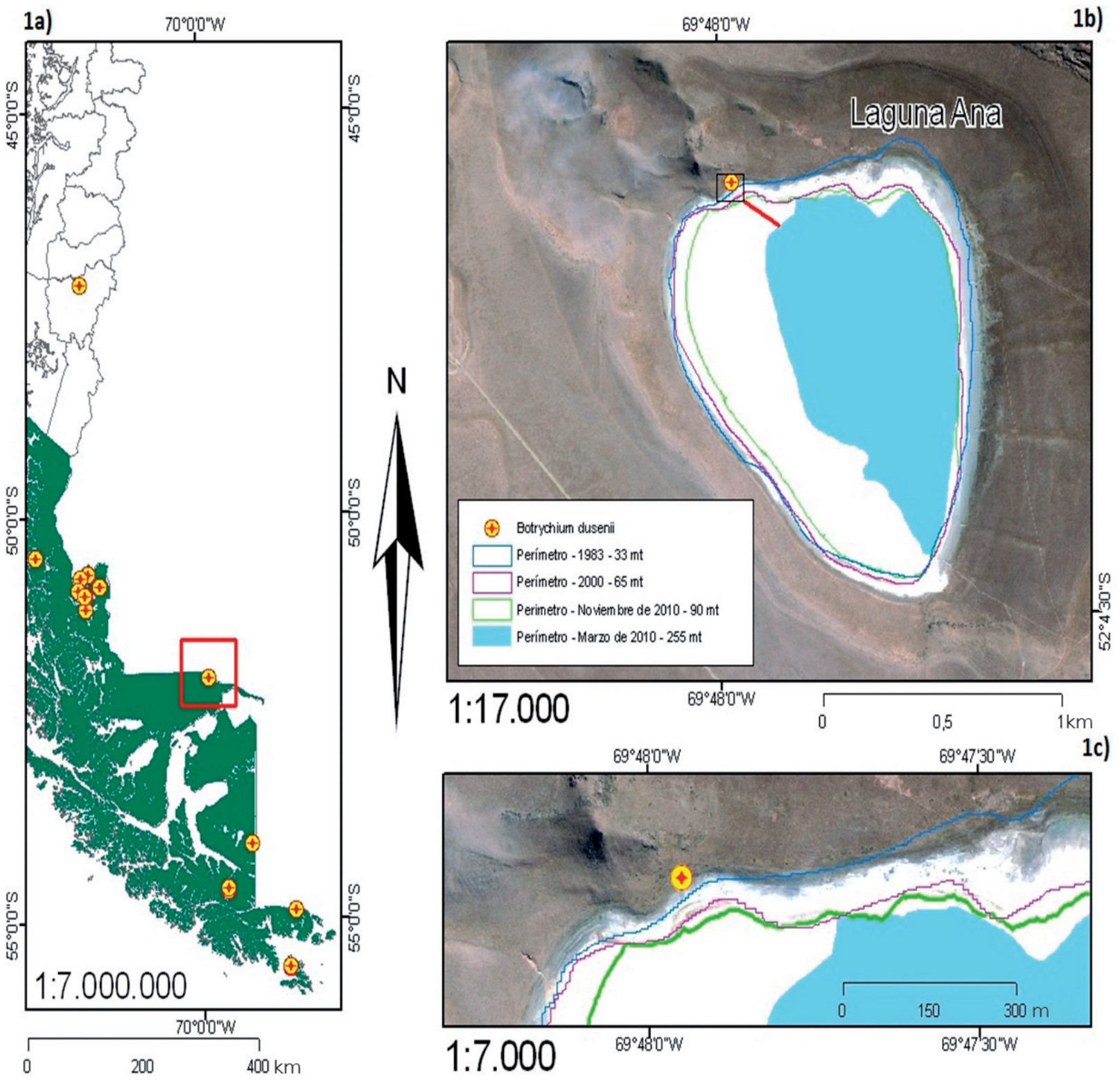

Figura 1. a) Mapa de distribución de Botrychium dusenii entre la XI y XII regiones, b) Laguna Ana y los perímetros descritos por el espejo de agua en cuatro momentos dentro de un periodo de 27 años (1983-2010), perímetros de un ciclo anual y el punto del hallazgo de la población de $B$. dusenii y c) Detalle de la ubicación de la población de $B$. dusenii respecto a los perímetros recabados en el estudio.

Figure 1. a) Región Aysén del General Carlos Ibáñez del Campo and Region de Magallanes, B. dusenii distribution map, b) Laguna Ana and the described areas by water surface at four points within a 27 years period (1983-2010), perimeter of a one year cycle and B. dusenii population point and c) Detail of the $B$. dusenii population location respect to the perimeters obtained in the study. 


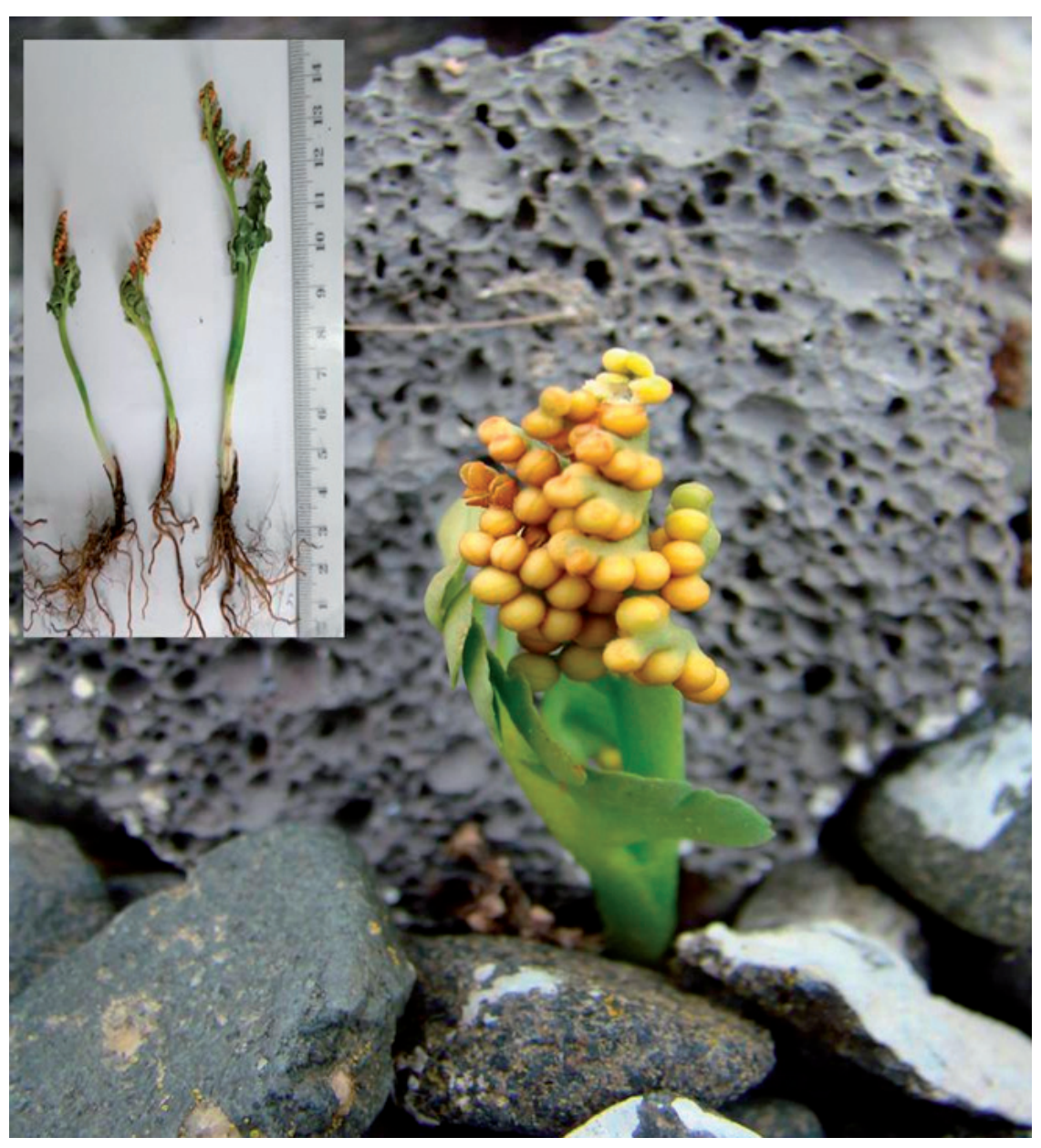

FIGURA 2. Botrychium dusenii y el ambiente donde se desarrolla, superficie caracterizada por ser arenosa, con rocas volcánicas y depósitos de sales cristalizadas. Imagen superior izquierda, muestras de individuos recolectados.

Figure 2. Environment where Botrychium dusenii is growing characterized by sandy surface with volcanic rocks and crystallized salts deposits. Top left, samples of individuals collected.

\section{Material estudiado}

Sierra de los Baguales, $50^{\circ} 45^{\prime} \mathrm{S}, 72^{\circ} 45^{\prime} \mathrm{W}$, Pisano y Cárdenas 4561 (HIP); Hostería las Torres, $51^{\circ} 00^{\prime} \mathrm{S}, 72^{\circ} 51^{\prime} \mathrm{W}$, Elvebakk 471 (HIP); Mirador del lago Nordenskjöld, 51 ${ }^{\circ} 02^{\prime} \mathrm{S}, 72^{\circ} 51^{\prime} \mathrm{W}$, Elvebakk 431 (HIP); Río Lazo, $51^{\circ} 05^{\prime} \mathrm{S}, 72^{\circ} 50^{\prime} \mathrm{W}$, Pisano 4145 (HIP); Sector Vicuña, lote

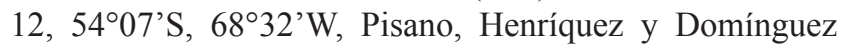
7286 (HIP); Isla Bayly, 55³8'S, 6767’ W, Dollenz 839 (HIP); Pisano 5170 (HIP); Cerro Guido, Pto. Natales, 5054'S, 72²8'W., Magens (CONC 27427); Isla Navarino, 54 $55^{\circ}$ 'S, 67³0'W, T-Tsujii (CONC 34204); Fiordo Parry, Bahía Cuevas, 5442'S, 69¹9'W, Pisano (CONC 68357); Magallanes. Ultima Esperanza, 50³0'S, 7400’W, C. Exss, (CONC 68358); Parque Nacional Torres del Paine, 5047'S, 7257'W, Arroyo y Squeo (CONC 86718); Cordillera del
Paine, 56 56' $\mathrm{S}, 7^{\circ} 00^{\prime} \mathrm{W}$, Von Bohlen y Cavieres (CONC 128372); Sierra del Toro, 51 ${ }^{\circ} 10^{\prime} \mathrm{S}, 72^{\circ} 50^{\prime} \mathrm{W}$, Arroyo, Von Bohlen y Cavieres (CONC 128514, 128544); Isla Bayly,

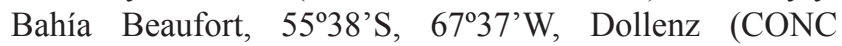
137886); Isla Bayly, Arenal, canal Victoria, 55³8'S, $67^{\circ} 37^{\prime} \mathrm{W}$, Pisano (CONC 141463); Tierra del Fuego, Sector Vicuña, Forestal Trillium, 5407'S, 68 $40^{\circ} \mathrm{W}$, Pisano, Henríquez y Domínguez (CONC 139764, 139765); Fiordo Parry, Parque Nacional Agostini, Caleta Alacaluf, 54 40'S, 69² $21^{\prime} \mathrm{W}$, Pisano, Henríquez y Domínguez (CONC 141553); Aysén. Capitán Prat, Colonia Norte, 47²10'S, $72^{\circ} 41^{\prime} \mathrm{W}$, Teneb (CONC 169202); Laguna Ana, Parque Nacional Pali Aike, 5203' S, 6976' W, Domínguez, Suárez y Bahamonde (CONC 172456). 
Gayana Bot. 68(2), 2011

Tabla I. Flora asociada a Botrychium dusenii en el Parque Nacional Pali Aike.

Table I. Botrychium dusenii associated flora in the Pali Aike National Park.

\begin{tabular}{|c|c|c|c|}
\hline FAMILIA & ESPECIES & FV & CoB. \\
\hline Rosaceae & Acaena magellanica (Lam.) Vahl & $\mathrm{Hc}$ & 0,14 \\
\hline Rosaceae & Acaena sericea J.Jacq. & $\mathrm{Hc}$ & 0,22 \\
\hline Cyperaceae & Amphiscirpus nevadensis (S.Watson) Oteng-Yeb. & $\mathrm{Hc}$ & 5,64 \\
\hline Ranunculaceae & Anemone multifida Poir. & $\mathrm{Hc}$ & 0,07 \\
\hline Apiaceae & Azorella fuegiana Speg. & $\mathrm{C}$ & 0,06 \\
\hline Apiaceae & Azorella monantha Clos & $\mathrm{C}$ & 0,11 \\
\hline Apiaceae & Azorella trifurcata (Gaertn.) Pers. & $\mathrm{C}$ & 1,22 \\
\hline Berberidaceae & Berberis empetrifolia Lam. & $\mathrm{Nf}$ & 0,07 \\
\hline Ophioglossaceae & Botrychium dusenii (H.Christ) Alston & $\mathrm{Hc}$ & 0,07 \\
\hline Calceolariaceae & Calceolaria uniflora Lam. & $\mathrm{Hc}$ & 0,04 \\
\hline Cyperaceae & Carex macrosolen Steud. & $\mathrm{Hc}$ & 0,22 \\
\hline Caryophyllaceae & Colobanthus quitensis (Kunth) Bartl. & $\mathrm{C}$ & 1,61 \\
\hline Caryophyllaceae & Colobanthus subulatus (d'Urv.) Hook.f. & $\mathrm{C}$ & 0,20 \\
\hline Brassicaceae & Draba verna $\mathrm{L}$. & $\mathrm{Te}$ & 0,09 \\
\hline Asteraceae & Erigeron patagonicus Phil. & $\mathrm{Hc}$ & 0,06 \\
\hline Poaceae & Festuca gracillima Hook. f. var. gracillima & $\mathrm{Hc}$ & 0,09 \\
\hline Asteraceae & Gamochaeta spiciformis (Sch.Bip.) Cabrera & $\mathrm{Hc}$ & 0,19 \\
\hline Poaceae & Hordeum comosum J.Presl & $\mathrm{Hc}$ & 7,83 \\
\hline Campanulaceae & Lobelia oligophylla (Wedd.) Lammers & $\mathrm{Hc}$ & 0,07 \\
\hline Asteraceae & Nassauvia darwinii (Hook. \& Arn.) O. Hoffm. \& Dusén & $\mathrm{Hc}$ & 0,07 \\
\hline Plantaginaceae & Plantago barbata G.Forst. subsp. barbata & $\mathrm{C}$ & 0,04 \\
\hline Poaceae & Poa alopecurus (Gaudich. ex Mirb.) Kunth subsp. alopecurus & $\mathrm{Hc}$ & 0,61 \\
\hline Poaceae & Poa compressa $\mathrm{L}$. & $\mathrm{Hc}$ & 0,17 \\
\hline Primulaceae & Primula magellanica Lehm. & $\mathrm{Hc}$ & 1,13 \\
\hline Poaceae & Puccinellia magellanica (Hook.f.) Parodi & $\mathrm{Hc}$ & 4,67 \\
\hline Poaceae & Rytidosperma virescens (E.Desv.) Nicora var. virescens & $\mathrm{Hc}$ & 0,12 \\
\hline Saxifragaceae & Saxifraga magellanica Poir. & $\mathrm{Hc}$ & 0,17 \\
\hline Asteraceae & Senecio miser Hook.f. & $\mathrm{C}$ & 0,11 \\
\hline Asteraceae & Senecio patagonicus Hook. \& Arn. var. patagonicus & $\mathrm{Nf}$ & 0,22 \\
\hline Asteraceae & Taraxacum officinale G.Weber ex F.H.Wigg. & $\mathrm{Hc}$ & 0,27 \\
\hline Valerianaceae & Valeriana carnosa $\mathrm{Sm}$. & $\mathrm{Hc}$ & 0,08 \\
\hline
\end{tabular}

Fv $=$ Forma de vida: $\mathrm{Hc}=$ Hemicriptófito, $\mathrm{C}=$ Caméfito, $\mathrm{G}=$ Geófito; Te = Terófito; Cob = Cobertura. $/$ Fv: Form life: Hc $=$ Hemicryptophyte, $\mathrm{C}=$ Chamephyte, $\mathrm{G}=$ Geophyte; $\mathrm{Te}=$ Terophyte; $\mathrm{Cob}=$ Coverage.

\section{AGRADECIMIENTOS}

Al proyecto: Estudio sobre el retroceso de las aguas superficiales del sector continental e insular, su incidencia en la producción ganadera y la importancia como parámetro del cambio climático en la Región de Magallanes, FIC Regional 2010.

\section{BIBLIOGRAFÍA}

Arroyo, M. T. K., C. von Bohlen, C. Cavieres \& C. Marticorena. 1992. Survey of the alpine flora of Torres del Paine National Park, Chile.Gayana Botánica 49:47-70.

Baeza, M., E. Barrera, J. Flores, C. Ramírez \& R. Rodríguez. 1998. Categorías de conservación de Pteridophyta nativas de Chile. Boletín del Museo Nacional de Historia Natural 47:23-46.

DollenZ, O. 1982. Estudios fitosociológicos en el archipiélago de Cabo de Hornos. III. Relevamientos en la isla Deceit. Anales Instituto Patagonia (Chile) 13:145-151.

Domínguez, E., E. Pisano \& O. Dollenz. 1999. Colonización Vegetal en el Glaciar Nueva Zelandia, Cordillera Darwin Chile. Anales Instituto Patagonia, Series Ciencias Naturales (Chile) 27: 7-16.

Domínguez E, C. Marticorena, A. Elvebakk \& A. Pauchard. 2004. Catálogo de la flora vascular del Parque Nacional Pali Aike, XII Región, Chile. Gayana Botánica 61(2): 6772.

Endlicher, W. \& A. SAntana. 1988. El clima del sur de la Patagonia y sus aspectos ecológicos. Un siglo de mediciones climatológicas en Punta Arenas. Anales Instituto Patagonia, Serie Ciencias Naturales 26:119 -128. 
Henríquez, J.M., E. Pisano \& C. Marticorena. 1995. Catálogo de la flora vascular de Magallanes (XII Región), Chile. Anales Instituto Patagonia. Serie Ciencias Naturales 23: 5-30.

Henríquez, J.M. 2002. Análisis de la flora vascular de valles glaciares de la región de Magallanes, Chile. Anales Instituto Patagonia, Serie Ciencias Naturales (Chile) 30: 25-40.

Looser, G. 1971. Botrychium lunaria (Pteridophyta) en Patagonia y Tierra del Fuego. Especie de área bipolar. Anales Instituto Patagonia (Chile) 2(1-2): 142-144.

Moore, D. M.1983. Flora Tierra del Fuego. A. Nelson-Missouri Botanical Garden, England-USA. 396 pp.

PisAno, E. 1974. Estudio ecológico de la región continental sur del área andino-patagónica. II. Contribución a la fitogeografía de la zona del parque Nacional Torres del Paine. Anales Instituto Patagonia 5(1-2): 59-104.

Pisano, E. 1977. Fitogeografía de Fuego-Patagonia chilena I. Las comunidades vegetales entre las latitudes $52^{\circ}$ y $56^{\circ}$ S. Anales Instituto Patagonia, Serie Ciencias Naturales 8: 121-250.
Pisano, E. 1980. Comunidades vegetales de la isla Hornos (archipiélago del Cabo de Hornos). Anales del Instituto de la Patagonia (Chile) 13: 125-143.

Rodríguez, R. 1995. Pteridophyta. En: C. Marticorena \& R. Rodríguez (eds.), Flora de Chile. Tomo I: Pteridophyta Gymnospermae, pp. 138-148. Universidad de Concepción, Concepción .Chile.

Rodríguez, R., D. Alarcón \& J. Espejo. 2009. Helechos Nativos del Centro y Sur de Chile. Guía de Campo. Ed. Corporación Chilena de la Madera, Concepción, Chile. 212 pp.

Saldivia, P. \& G. Rojas. 2006. Valeriana moyanoi Speg. (Valerianaceae), Nuevo registro para la Flora de Chile. Gayana Botanica 63(2): 183-186.

Zuloaga, F.O., O. Morrone \& M.J. Belgrano. 1994 en adelante. Catálogo de Plantas Vasculares del Cono Sur. Website http://www.darwin.edu.ar/Proyectos/FloraArgentina/ FA.asp. Actualizado a Enero 2009. Consulta: Diciembre 2010.

Recibido: 25.03.11

Aceptado: 02.08.11 